\title{
Adult Emergency Department Patients with Sickle Cell Pain Crisis: Results from a Quality Improvement Learning Collaborative Model to Improve Analgesic Management
}

\author{
Paula Tanabe, PhD, RN, MSN, MPH, John W. Hafner, MD, MPH, Zoran Martinovich, PhD, \\ and Nicole Artz, MD \\ Schools of Nursing and Medicine, Duke University, (PT) Durham, NC; Department of Surgery - \\ Emergency Medicine, University of Illinois College of Medicine at Peoria, and OSF Saint Francis \\ Medical Center, (JWH) Peoria, IL; Division of Psychology, Mental Health Services and Policy \\ Program, Feinberg School of Medicine, Northwestern University, (ZM) Chicago, IL; Loyola \\ University Health System, (NA) Chicago, IL Previously: University of Chicago (NA), Chicago, IL
}

\begin{abstract}
Objectives-The aim of this study was to 1) estimate differences in pain management process and patient-reported outcomes, pre- and post-implementation of analgesic protocols for adults with sickle cell disease (SCD); and 2) examine the effects of site and visit frequency on changes in pain scores and time to analgesic.
\end{abstract}

Methods-A multi-center, prospective, longitudinal study enrolled patients from three academic medical centers between October 2007 and September 2009. All ED patients 18 years or older with a chief complaint of a sickle cell pain episode were enrolled. Sites formed a SCD quality improvement team and implemented standard nurse-initiated emergency department (ED) analgesic protocols; outcomes were compared between study periods defined as pre- and postimplementation of protocols. Medical record review was conducted to measure time to administration of initial analgesic, opioids used, route of opioid administration, the change in pain scores from arrival to discharge (negative numbers reflect a decrease in pain scores), and the number of ED visits per individual patient during the study period at each site. On day seven after the ED visit, a follow-up phone interview was conducted. Patients were queried about their ED pain management using a scale from 1-10 ( $1=$ outstanding, $10=$ worst $)$. Descriptive statistics are used to report the results. Ordinary least squares regression models were constructed to measure the effect of time period, site, and number of visits per patient on change in pain score.

Results-During the study period, 342 unique patients (57\% female, mean age 32 years, SD \pm 11 years) were enrolled and had a total of 2,934 visits. There was no difference in time to administration of the initial analgesic between study periods. Overall, there was a significant decrease in pain scores from arrival to discharge between the pre- and post-intervention study periods: the average difference in arrival to discharge pain scores $(\mathrm{cm})$ was greater during the post-implementation period than during the per-intervention period $(-4.1 \mathrm{vs}-3.6, \mathrm{t}=2.6, \mathrm{p}<$ 0.01 ). Site 1 had significant improvement between study periods (mean difference $=-0.87, \mathrm{t}=$ $2.63, \mathrm{p}<0.01 ; \mathrm{F}=14.3, \mathrm{p}<0.01$ ). Patients with few ED visits (one to six annual visits, mean difference $-1.55, \mathrm{t}=2.1, \mathrm{p}=0.04$ ) and those with frequent $\mathrm{ED}$ visits (seven to 19 annual visits, mean difference $-1.65, \mathrm{t}=3.52, \mathrm{p}<0.01$ ) had a significant decrease in pain scores as compared to

Corresponding author: Paula Tanabe, PhD, RN, MSN, MPH Duke University Clipp Building, Office \#1001, Durham, NC B: 919-613-6038; F: 919-681-8899; C: 312-502-0185 paula.tanabe@ duke.edu.

Presentation: Society for Academic Emergency Medicine, Boston MA, June 2011

The authors have no further disclosures or conflicts of interest to report. 
patients with very frequent ED visits ( $>19$ visits). There was an overall decrease in the use of morphine sulfate and increase in the use of hydromorphone $\left(x^{2}=105.67, p<0.001\right)$ between study periods and a significant increase in the use of oral and subcutaneous routes, with a corresponding decrease in the intravenous route $\left(x^{2}=13.67, \mathrm{p}<0.001\right)$. There were no statistically significant differences in patient-reported satisfaction with the attempt to manage pain in the ED between study periods $(\mathrm{p}=0.54)$.

Conclusions-While the use of a learning collaborative and implementation of nurse-initiated analgesic protocols was not associated with improvement in time to administration of the initial analgesic, improvements in the decrease in the arrival to discharge pain score, and increased use of hydromorphone and the subcutaneous route, were noted in adults with SCD in the ED.

\section{INTRODUCTION}

Sickle cell disease (SCD) is a genetic disorder of the hemoglobin affecting approximately 100,000 primarily African Americans in the United States. ${ }^{1}$ The lifespan for individuals with SCD remains much shorter for males and females (42 and 48 years respectively), when compared to the average lifespan for African American individuals in the United States (71 and 77 years). ${ }^{2}$ This shortened lifespan is due to a plethora of serious complications associated with the disease. These include but are not limited to stroke, sepsis, acute chest syndrome, pulmonary embolus, pulmonary hypertension, and renal failure. Yet, the most common reason for emergency department (ED) visits is evaluation and treatment of a vasoocclusive crisis (VOC). Associated pain requires rapid evaluation and treatment to 1) ensure other complications are not present, and 2) treat and prevent the pain from worsening and possibly avoid the need for a hospital admission.

Despite the benefit of rapid analgesic treatment, time to treatment remains prolonged and has been found to be longer than patients with renal colic. ${ }^{3,4}$ EDs face daily crowding that challenges the ability to provide rapid evaluation for specific life-threatening conditions and conditions, with indicators tied to reimbursement based upon time delivered interventions. These situations challenge the ability to rapidly place and evaluate all ED patients. ${ }^{5}$ Another factor that may contribute to delays in the evaluation and treatment of the patient with SCD is the frequently held belief that most patients with SCD are "drug seeking" and addicted to opioids, despite a lack of evidence to support this belief. In a survey of emergency medicine resident physicians and nurses, both groups reported a higher perception of addiction among SCD patients with pain when compared with all ED patients with pain.

An additional and very important barrier to treating pain in a timely fashion is the small number of SCD patients with very frequent ED visits. National data have identified that most patients with SCD have between one to two ED visits per year; however, a very small number of adults with SCD have frequent (more than three visits per year) ED visits. ${ }^{7-10}$ Reasons for this high use are not fully understood, but the following variables have been found to be associated with higher ED and hospital utilization: more severe disease and disease-related complications, worse pain, higher white blood cell counts, lower hemoglobin levels, asthma, being publically insured, 18 to 30 years old, and an inability to obtain followup appointments with hematology. ${ }^{7,9,11-13}$ Due to the frustration often expressed by clinicians when providing care to patients with frequent visits, we felt it was important to analyze this sub-group to determine if their analgesic management different from that of adult SCD patients with few ED visits.

While improving time to pain relief is the most meaningful outcome for patients, there are other outcomes more directly in the control of emergency clinicians (physicians and nurses) that can also be targeted to improve analgesic management. These outcomes include the 
patients' perception of pain management, change in pain scores during ED stay, and use of more appropriate agents and routes of administration.

In an effort to improve pain management for adults with SCD in EDs, our study team designed and implemented a research project with a strong quality improvement (QI) model. We previously reported study methodology and baseline findings, prior to the implementation of analgesic protocols. The aim of this study was to 1) estimate differences in pain management process and patient reported outcomes, pre- and post-implementation of analgesic protocols for adults with SCD; and 2) to examine the effects of site and visit frequency on changes in pain scores and time to analgesic.

\section{METHODS}

\section{Study Design}

Study methods have been previously reported but will be briefly summarized. ${ }^{14} \mathrm{We}$ conducted a multi-site prospective, longitudinal cohort study using a quality improvement framework with three EDs in Illinois. This design was selected to evaluate real-world practice. The effects of analgesic protocols on study outcomes were evaluated using a preand post-implementation design. The pre-implementation period was 10 months at study sites 1 and 2, and three months at study site 3. Each study site obtained institutional review board approval, and patients provided informed consent prior to participation in follow-up interviews for the duration of the project. IRBs from each site granted a waiver of consent to obtain medical record data for all SCD visits during the study period.

\section{Study Setting and Population}

All three sites were academic medical centers with emergency medicine residency programs. Two sites (\#1 and \#3) were located in an urban area, and the third site (\#2) was located in a mixed urban/rural area. Study sites reported a combined annual ED census of approximately 169,000 annual visits.

All adult ED visits with a chief complaint of VOC (identified by the International Classification of Disease-Ninth Revision) were included in the medical record review portion of the study. All adult patients (ages $\geq 18$ ) with a chief complaint of a VOC were eligible for participation in the follow-up interview. Individual patients were excluded from participation in the interview portion of the study if they did not speak English or were unable to provide follow-up contact information. Ten patients were invited to participate in one interview every three months.

\section{Study Protocol}

Study Site, QI Teams Meetings—Each site was asked to form a multi-disciplinary QI team affiliated with the project. The purpose of the team was to develop, implement, and evaluate analgesic protocols. Site 1 conducted monthly meetings with emergency physicians (EPs), nurses, and a hematologist throughout the project. Over time, it became more difficult to continue to engage EPs in the process, although monthly meetings were still held. Site 2's QI team included an EP champion who was also the local site primary investigator (PI), an ED resident physician, an ED charge nurse, an ED nurse educator, an RN clinical research coordinator, an ED social worker, and a six sigma quality assurance RN. The analgesic protocol was reviewed by a hematologist. Initially, the team met monthly, and then quarterly to discuss the protocol, individual patients, and use patterns; provide feedback data to the local team members and the ED staff; discuss barriers in the ED; and make further revisions to the protocol. Site 3's QI team members included the medical director for the adult sickle cell disease program, ED charge nurse, SCD nurse practitioner, and SCD and ED social 
workers. Initially, meetings were held monthly, and later quarterly to review barriers to protocol implementation and to revise the protocol. There was significant organizational change and turnover of ED nursing leadership at Site 3, making it difficult to continue to conduct QI meetings at this site.

Analgesic Protocols-Each site was allowed to develop slightly different analgesic protocols. As this was not a randomized controlled trial, it was not possible to, nor did the study team wish to "dictate" a specific protocol to any site. However, members of the study team from each of the sites participated in monthly calls and collaborated on protocol development. Two study sites allowed nurses to administer subcutaneous (SC) opioids in the waiting room. All three protocols included the use of intravenous (IV) hydromorphone or morphine sulfate, promoted the use of the SC route vs. intramuscular (IM) when IV access was difficult, and included re-assessment of oxygenation and sedation status prior to readministration of opioids. Re-assessment and re-administration of additional doses was allowed within the protocol using 15 to 20 minute intervals for the first three doses. All sites implemented nurse-initiated protocols that allowed ED nurses the autonomy to administer the first analgesic dose prior to physician evaluation, in accordance with the protocol. All three protocols underwent rigorous review by their respective departments, their pharmacy and therapeutics committees prior to implementation.

Enrollment and Patient Interviews-The project was implemented between October 2007 and September 2009. All patients with a chief complaint of a VOC were approached by a research assistant either during an ED visit or hospitalization and invited to participate. Research assistants at each site were trained by the site PI. Site PIs were trained by the project PI (PT) prior to initiation of the study. Patients who agreed to participate signed a written consent form agreeing to be contacted for follow-up interviews after the ED visits for the duration of the project (three years); they were informed they could withdraw from the study at any time. Each site conducted a total of 10 interviews per quarter. The rationale for this number of interviews was 1) the QI methodology uses a small sample, 2) site 2 had a small overall number of patients and it would be undesirable to continually interview the same patients, and 3) a sample of 10 interviews per quarter/site is sufficient to answer the study questions. Subjects were eligible to participate in one interview every three months. Interviews were conducted between 7 and 14 days after the ED visit and focused on the patients' perception of ED pain management. Interviews were conducted by phone unless the patient was hospitalized and well enough to participate in an in-person interview. No interviews were conducted during the ED visits.

Structured medical record review-All ED visits with a chief complaint of a VOC were included in the analysis. To evaluate inter-rater reliability of key outcome variables, we used SPSS to generate a random sample (IBM SPSS, Armonk, NY). The PI abstracted key variables for the selected sample. An initial random sample of 5\% of visits yielded sufficiently high reliability estimates so that further sampling for reliability estimation was deemed unnecessary. The lowest reliability (drug name) was quite high (kappa $=0.88)$ and other items were more reliable (time to initial analgesic, Cronbach's alpha $=0.99$, and route of initial analgesic, kappa $=0.98$ ).

Study variables-Patient characteristic variables included self-reported age, sex, and race. Differences in pain management process outcomes included the following: 1) time in minutes from arrival at triage to administration of first analgesic, 2) change in pain scores between arrival and within 45 minutes of leaving the ED (admitted or discharged home) when documented in the medical record $(0-10 \mathrm{~cm}, 10=$ worst pain imaginable $)$, and 3$)$ change in initial analgesic agent and route of administration. The change in pain score was 
created as a new variable by subtracting the arrival pain score from the last pain score documented within 45 minutes of leaving the ED (admitted or discharged). Analgesic agents used during the study period included morphine sulfate (MS), hydromorphone, meperidine, fentanyl, hydrocodone/acetaminophen, MS and ibuprofen or ketorolac, and hydromorphone and ibuprofen or ketorolac. Routes of administration were coded as intravenous (IV), subcutaneous (SC), intramuscular (IM), or oral (PO).

The difference in patient-reported perception of how pain was managed in the ED pre- and post-implementation of analgesic protocols was evaluated by the following question during the follow-up interview: "Please rate how well you believe the ED doctors and nurses tried to manage your pain using a scale from $1-10,1=$ outstanding, $10=$ worst." This reverse scale was used because patients on the advisory panel for the project advised us that patients equate " 10 " with bad and " 1 " with good. In order to examine the effects of visit frequency on changes in pain scores and time to analgesic, the total number of ED visits for the entire study period, per patient at each study site, was calculated using medical record review. The total number of visits per patient was later categorized into three categories: low (one to six visits), medium (seven to 19 visits), and high (20 or more visits).

\section{Data Analysis}

All data were entered directly by each study site into a web-based SNAP database (Snap Surveys, Ltd., London, UK). Data were exported into and analyzed using IBM SPSS (release 19.0.0). Means and standard deviations (SDs) are used to report normally distributed data, and medians with inter-quartile ranges (IQRs) are used to report data for skewed distributions. Primary dependent variables were time to analgesic and change in pain score between admission and discharge. The primary unit of comparison for outcome variables was the preversus post-implementation study period samples. Ordinary least squares (OLS) regression models were constructed with utilization group (i.e., three categories defined by visits/patient) and study period sample (pre- vs. post-implementation) as crossed between subject factors to predict within-visit pain change and time to analgesic. Similarly structured models were constructed using site and study period sample as crossed factors predicting within-visit pain change and time to analgesic. The linear model equations for four models evaluated are:

$$
\begin{array}{ll}
\text { Model 1: } & T A=B_{0}+B_{1} \text { Period }+\sum_{i=1}^{2} B_{2 i} \text { Site }_{i}+\sum_{i=1}^{2} B_{3 i} \text { Period } \times \text { Site }_{i}+\varepsilon \\
\text { Model 2: } & T A=B_{0}+B_{1} \text { Period }+\sum_{i=1}^{2} B_{2 i} \text { Site }_{i}+\sum_{j=1}^{2} B_{3 i} \text { Visit }_{i}+\sum_{j=1}^{2} B_{4 j} \text { Period }^{2} \times \text { Visit }_{j}+\varepsilon \\
\text { Model 3: } & P C=B_{0}+B_{1} \text { Period }+\sum_{i=1}^{2} B_{2 i} \text { Site }_{i}+\sum_{i=1}^{2} B_{3 i} \text { Period } \times \text { Site }_{i}+\varepsilon \\
\text { Model 4: } & P C=B_{0}+B_{1} \text { Period }+\sum_{j=1}^{2} B_{2 j} \text { Visit }_{j}+\sum_{j=1}^{2} B_{3 j} \text { Period } \times \text { Visit }_{j}+\varepsilon
\end{array}
$$

where TA = time to analgesic, $\mathrm{PC}=$ pain change, Period = pre- vs. post-time sample, Site (three levels, dummy coded), and Visit = (three levels, dummy coded), and where $\mathrm{i}$ and $\mathrm{j}$ subscripts denote separate terms for each dummy code (two for site and visit, respectively)

These models are essentially conventional ANOVA models with crossed factors; however, for analyses of the effect of utilization group on time to analgesic (model 2), site was included as a three-level additive dummy-coded control variable (due to substantial site differences in time to analgesic means). Site was investigated as a control variable for pain change analyses (and excluded, since inclusion did not alter the results). Due to distribution skew, time to analgesic was log-transformed and analyses were conducted on the 
transformed roughly symmetric distribution for Models 1 and 2. After transformation, all significant effects remained significant, and non-significant effects remained unchanged. Only analyses on the not-transformed dependent variable are included in this article. Since all predictors were categorical, the present regression models reduce to ANOVA models. Because cell sample sizes were substantial (far above 30), the distribution of cell means is nearly normally distributed (central limit theorem), and the present analyses may be considered quite robust with respect to violations of normality of within-cell residuals (leading to a consistency of inferential results with vs. without transformations of time to analgesic data). Pain change distributions were more nearly symmetric and cell variability was plausibly homogeneous for Models 3 and 4 .

Changes in median time to initial analgesic and differences in pain scores (from arrival to discharge) between pre- and post-implementation periods were also evaluated via an independent Hodges-Lehman 95\% CI for independently sampled medians with associated non-parametric inferential tests. Confidence intervals were estimated for the full sample, separately within each site, and separately for each study visit utilization group as defined above.

Changes in percent use of analgesic agents and specific routes of administration pre- vs. post-implementation of analgesic protocols were estimated, overall and separately for each study site. Rates were compared via chi-square tests of independence. ANOVA was used to analyze differences in the number of ED visits per individual patient at each site over the duration of the entire study period, with planned pairwise comparisons of sites (unadjusted ttests).

Differences in patient-reported satisfaction scores in the clinicians' attempt to manage pain between the pre- and post-implementation periods was analyzed using an independent samples t-test.

\section{RESULTS}

A total of 342 patients participated in the study (site 1, $\mathrm{n}=99$; site $2, \mathrm{n}=31$; site $3, \mathrm{n}=212$ ). Table 1 reports subject characteristics per study site. Figure 1 depicts boxplots of the number of visits per patient for the three study sites. Site 1 had 959 total ED visits, site 2 had 807, and site 3 had 1,169, for a total of 2,935 ED visits from 342 patients across all sites combined. The mean number of visits per patient was $8.6(\mathrm{SD} \pm 19.4)$. The site 2 mean number of visits per patient was significantly higher than the site 1 average $(\mathrm{t}(339)=4.29, \mathrm{p}$ $<0.001)$, and the site 2 average was significantly higher than the site 3 average $(\mathrm{t}(339)=$ $5.76, \mathrm{p}<0.001$ ). The median visits per patient from sites 1,2 , and 3 were 2 (IQR 1 to 10), 6 (IQR 2 to 32), and 2 (IQR 1 to 6), respectively.

\section{Time to analgesic administration}

Table 2 describes median differences in the time to the administration of the initial analgesic at all sites between pre- and post-implementation of analgesic protocols. The average time to analgesic administration was greater during the post-implementation period than the preimplementation period ( 131 minutes vs 114 minutes, $\mathrm{t}(2871)=3.40, \mathrm{p}=0.001)$. However, when including site and study period as crossed factors predicting time to analgesic (Model 1 ), only the site main effect was significant (Table 3). A modest study period effect was present within site 2 , with time to analgesic increasing from 69 to 78 minutes $(\mathrm{t}(802)=2.76$, $\mathrm{p}=0.006$ ). However, the full sample study period effect was primarily attributable to the site differences in time to analgesic and site differences in sample size distributions between pre- and post-implementation periods (i.e., confounding study period effects with site main 
effects). The difference in samples size distribution was primarily due to a shortened preimplementation period for site 3 .

Table 3 (Model 2) included visit group and study period as crossed factors, while additively controlling for site. Neither visit group, study period, nor the interaction were significant predictors of time to analgesic.

\section{Change in Pain Scores, Arrival to Discharge}

Table 4 presents median differences in arrival to discharge pain scores in the pre- to postimplementation periods for the entire sample, and by site and visit category. There were 295 ED visits with complete data available to calculate the change in pain score variable in the pre-intervention period, and 458 in the post-intervention period. The average difference in arrival to discharge pain scores $(\mathrm{cm})$ was greater during the post-implementation period than during the pre-implementation period $(-4.1 \mathrm{vs}-3.6,(\mathrm{t}=2.6, \mathrm{p}=<0.01)$. When including site and study period as crossed factors predicting change in pain scores (Model 3 ), study period was no longer significant. A strong effect was attributable to site differences in change in pain score by time period (Table 5, Figures 2A). Site 1 had significant improvement between the pre- and post-implementation study periods.

In Model 4, visit group and study period were included as crossed factors - both main effects and interaction were significant (Table 5, Figure 2B). The number of visits was associated with a decrease in pain score between study periods with a significant decrease found in patients in the 1 to 6 visit category and the 7 to 19 visit category. Results from the regression models demonstrate patients from site 1 , and patients with fewer visits, experienced the largest decrease in pain scores from the pre- to the post-implementation period; the largest effect was seen in patients treated at site 1.

\section{Patient reported outcomes}

There were no statistically significant differences in patient-reported satisfaction with the attempt to manage pain in the ED between study periods $(\mathrm{p}=0.54)$. Overall mean satisfaction levels were 3.4 ( $\mathrm{SD} \pm 2.9)$ in the pre-implementation period $(\mathrm{m}=81$ interviews) and $3.2(\mathrm{SD} \pm 2.4)$ in the post-implementation period $(\mathrm{n}=126$ interviews). A low score represented a high level of satisfaction, indicating patients were moderately satisfied with the ED's management of their pain.

\section{Analgesic agents and Routes}

Table 6 reports changes in analgesic agents and routes used between the pre- and postimplementation study periods. There was an overall decrease in the use of morphine sulfate and increase in the use of hydromorphone $\left(x^{2}=105.67, \mathrm{p}<0.001\right)$. Sites 2 and 3 had the largest increase in the use of hydromorphone. There was a significant increase in the use of $\mathrm{PO}$ and SC routes, with a corresponding decrease in the IV route $\left(\mathrm{x}^{2}=13.67, \mathrm{p}<0.001\right)$. The largest differences were attributable to an increase in use of the SC route at site 1.

\section{DISCUSSION}

We report data from the first prospective, multi-center, QI study aimed at improving analgesic management for adults with SCD in the ED setting. Previous single-site studies have reported changes in ED and hospital utilization patterns after instituting standing analgesic orders for all adults with SCD, or individualized plans for SCD patients with frequent use. ${ }^{15-17}$ However, these studies did not evaluate the effect of protocols on pain management. Rapid assessment and treatment of pain for patients with SCD is advocated by the American Pain Society; the National Institutes of Health; National Heart, Lung, and 
Blood Institute, Division of Blood Diseases; and the Sickle Cell Society in the United Kingdom. ${ }^{18-20}$ These guidelines suggest patients receive an initial analgesic within 15 to 30 minutes of arrival. While this timeframe may be challenging, the recommendations are supported by findings from the Cooperative Study of Sickle Cell Disease (CSSCD), which followed 3,764 patients with SCD to determine life expectancy and cause of death. ${ }^{21}$ Thirtythree percent of the patients that died did so during an acute VOC (45 deaths, 20 complicated by acute chest syndrome, 25 uncomplicated VOC), while other deaths were attributed to acute chest syndrome $(n=9)$, renal failure $(n=22)$, infection $(n=13)$, and stroke $(n=15)$. The most frequent reason for ED visit is an acute VOC; however, patients have a severely shortened life expectancy and may be also be experiencing other complications that require rapid evaluation and treatment.

From a patient perspective, the time to receiving the first analgesic is probably the most important outcome. Crowding is prevalent throughout the United States and presents an important barrier to timely analgesic administration for all patients presenting with acute pain. Protocols at two of the study sites allowed nurses to initiate subcutaneous doses in the waiting room if a treatment bed was not immediately available. Despite this autonomy, we did not find significant improvement in time to initial analgesic at any of the three sites. Nurses at both sites anecdotally reported several barriers to providing opioids in the waiting room, including the difficulty of leaving a busy triage area to obtain opioids from the medication administration area in the main treatment area.

Nurses also expressed reluctance on the ability to monitor patients in the waiting room after administering an opioid. All three site protocols allowed for the first analgesic to be provided by the registered nurse prior to the physician evaluating the patient, if specific contraindications were not met. Despite the standing orders, many nurses anecdotally reported reluctance to administer an opioid prior to physician evaluation. Further exploration is necessary to help understand how nurses can be made more comfortable with standing analgesic orders. In order for EDs to provide more rapid analgesia, nurses should be given the autonomy to follow standing orders, and must be comfortable with these orders. We also believe the lack of improvement in the time to analgesic outcome was attributable to the challenges experienced at different sites. Site 1 had the highest level of acceptance among both EPs and nurses. We believe this was due to the strong participation in the site's QI team and frequency of meetings. Site 3 experienced large organizational restructure with significant ED nursing leadership turnover. We believe an additional reason we did not find more promising results was the inability of any of the sites to incorporate QI measures into their ED QI plans in a formal measure.

Finally, it is also not surprising that time to analgesics did not improve overall because crowding continued to be a significant challenge at all three study sites. Overall time to initial analgesic is a very challenging outcome to alter, given the continued rate of crowding in most EDs. Arendts et al. found several factors that contribute to delays to receiving opioid analgesia in EDs. ${ }^{22}$ These factors included having a medical vs. a trauma diagnosis, being female, receiving a lower priority triage code, and being taken care of by a more junior physician. SCD is a medical disease and we have shown that SCD patients who received a lower priority triage score experienced longer times to receiving an initial analgesic. While all patients with severe pain remain a high priority, we believe that SCD patients should be at the top of the list due to the other serious life-threatening complications. Anecdotally, patients report that if their pain is quickly and aggressively managed, they are more likely to be able to be discharged home. Further study is warranted.

While administering analgesics in a timely fashion is an important and proxy outcome often used to assess pain relief, there are other important outcomes that can be measured to assess 
pain management outcomes. In anticipation of the challenges in improving time to the provision of the initial analgesic, additional a priori analyses were planned, which included examining the change in pain scores between arrival to discharge, and the patients' reports of the ED clinicians' attempts to manage their pain. We chose to measure the absolute change in score vs. the proportion of change because we felt this was a good representation of change in pain score. We found significant improvements in the overall change in pain scores from arrival to within 45 minutes of leaving the ED. These changes were attributable to site differences (Site 1) and visit frequency (patients with less than 20 ED visits during the study period). We explain these differences by the good functioning of the QI team at Site 1 , although it is possible other unknown variables may explain the difference. Patients with more than $20 \mathrm{ED}$ visits did not experience a significant improvement in arrival to discharge pain scores from the pre- to post-implementation periods. This could be attributable to the frustration experienced by many ED clinicians when caring for adults with SCD on a very frequent basis, or a greater level of opioid tolerance in these patients making it more difficult to achieve control of their pain. Patient reports of satisfaction with the clinicians' attempts to manage their pain also did not change from the pre- to postimplementation periods; however, the levels indicated moderately high satisfaction during both periods. When patients were asked this question during the follow-up interviews, anecdotal comments indicated that while often frustrated with waiting, they believed that clinicians were generally trying to manage their pain. The study team was somewhat surprised by this finding, and by the anecdotal comments that indicated patients in general had a very low level of expectations.

A major focus of the pain management protocols was promoting the use of the subcutaneous route when IV access was difficult. There were several reasons for promoting this change. Frequently, significant delays occur when nurses continue to attempt to obtain IV access. In these situations, nurses will sometimes administer opioids using the IM route, which is associated with tissue damage. The SC route is routinely used with oncology patients, and is advocated by the American Pain Society, National Institutes of Health, National Heart, Lung, and Blood Institute, Division of Blood Diseases, and the Minnesota Department of Public Health Sickle Cell Disease Emergency Guidelines. ${ }^{18,19,23}$ There was a significant increase in the use of the SC route at two of the three study sites. Neither EPs nor nurses were initially aware of the rationale or ability to use the SC route when IV access was difficult; clinicians typically resort to the IM route out of habit. Oral agents are not preferred because of the delay in onset of effect, and many patients have already taken oral opioids prior to coming to the ED. Education regarding the rationale and methods of administration was received very positively. Finally, there was an overall increase in the use of hydromorphone with a corresponding decrease in the use of morphine sulfate. Either agent is recommended. The main difference in these agents is the equianalgesic dosing. Hydromorphone $1.5 \mathrm{mg}$ intravenously is equal to approximately $10 \mathrm{mg}$ of morphine sulfate. ${ }^{24}$ Use of either agent is acceptable; however, because many clinicians do not appreciate the equianalgesic dosing differences between MS and hydromorphone, patients often receive a high equianalgesic dose of hydromorphone, which may account for better pain relief.

\section{LIMITATIONS}

This was not a randomized controlled trial. A quality improvement and research project was used to evaluate the success at each center. Protocols were slightly different, and we could not control for other very significant changes that took place at one of the study sites. We conducted a structured medical record review, which is subject to the limitations of accurate documentation. However, we did perform inter-rater reliability checks of the data, and historically, nurses are very accurate about documenting medications. We analyzed the 
difference in pain scores only for those visits in which there were both an arrival pain score, and a discharge pain score within 45 minutes of arrival, leaving significant missing data. The time to administration of the initial analgesic analysis was limited by the very small number of patients enrolled at Site 3 in the pre-implementation study period ( 3 months vs. 10 months at the other sites), which influenced findings. Finally, improvements in arrival to discharge pain scores may have been caused by an overall increase in the use of hydromorphone.

\section{CONCLUSIONS}

The use of quality improvement teams and implementation of nurse-initiated analgesic protocols was not associated with improvement in time to administration of the initial analgesic, but was associated with improvement in the decrease in pain score, and increased use of hydromorphone and the subcutaneous route of medication administration in adults with sickle cell disease in the emergency department.

\section{Acknowledgments}

The authors would like to thank Elena Zvirbulis, project coordinator and, Alex Chiu, Christiana Chukwu, Matt Gilbert, Julie Stevens, Autumn Stevenson, and Kristen Turner for assistance with data abstraction.

Financial support: This project was supported by a research grant from the Mayday Fund from the University of Chicago. Dr. Tanabe also received support from the NIH, K23NR010940.

\section{REFERENCES}

1. Hassell KL. Population estimates of sickle cell disease in the U.S. Am J Prev Med. 2010; 38:S51221. [PubMed: 20331952]

2. Kochanek, KD.; Xu, J.; Murphy, SL.; Minino, AM.; Kung, M. Deaths: preliminary data for 2009. National vital statistics report. 2011.

3. Tanabe P, Myers R, Zosel A, et al. Emergency department management of acute pain episodes in sickle cell disease. Acad Emerg Med. 2007; 14:419-25. [PubMed: 17389246]

4. Lazio MP, Costello HH, Courtney DM, et al. A comparison of analgesic management for emergency department patients with sickle cell disease and renal colic. Clin J Pain. 2010; 26:199_ 205. [PubMed: 20173433]

5. Institute of Medicine. [Accessed Jan 21, 2012] The future of emergency care in the united states health system. Available at: http://www.iom.edu/Activities/Quality/emergencycare.aspx

6. Waldrop R. Health professional perceptions of opioid dependence among patients with pain. Am J Emerg Med. 1995; 13:529-31. [PubMed: 7662056]

7. Lanzkron S, Carroll CP, Haywood C Jr. The burden of emergency department use for sickle-cell disease: an analysis of the national emergency department sample database. Am J Hematol. 2010; 85:797-9. [PubMed: 20730795]

8. Brousseau DC, Owens PL, Mosso AL, Panepinto JA, Steiner CA. Acute care utilization and rehospitalizations for sickle cell disease. JAMA. 2010; 303:1288-94. [PubMed: 20371788]

9. Carroll CP, Haywood C Jr, Fagan P, Lanzkron S. The course and correlates of high hospital utilization in sickle cell disease: evidence from a large, urban Medicaid managed care organization. Am J Hematol. 2009; 84:666-70. [PubMed: 19743465]

10. Kauf TL, Coates TD, Huazhi L, Mody-Patel N, Hartzema AG. The cost of health care for children and adults with sickle cell disease. Am J Hematol. 2009; 84:323-7. [PubMed: 19358302]

11. Aisiku IP, Smith WR, McClish DK, et al. Comparisons of high versus low emergency department utilizers in sickle cell disease. Ann Emerg Med. 2009; 53:587-93. [PubMed: 18926599]

12. Wolfson JA, Schrager SM, Coates TD, Kipke MD. Sickle-cell disease in California: a populationbased description of emergency department utilization. Pediatr Blood Cancer. 2011; 56:413-9. [PubMed: 21225920] 
13. Frei-Jones M, Field J, Debaun M. Risk factors for hospital re-admission within 30 days: a new quality measure for children with sickle cell disease. Pediatr Blood Cancer. 2008; 52:481-5. [PubMed: 19058209]

14. Tanabe P, Artz N, Courtney DM, et al. Adult emergency department patients with sickle cell pain crisis: a learning collaborative model to improve analgesic management. Acad Emerg Med. 2010; 17:399-407. [PubMed: 20370779]

15. Givens M, Rutherford C, Joshi G, Delaney K. Impact of an emergency department pain management protocol on the pattern of visits by patients with sickle cell disease. J Emerg Med. 2007; 32:239-43. [PubMed: 17394984]

16. Friedman EW, Webber AB, Osborn HH, Schwartz S. Oral analgesia for treatment of painful crisis in sickle cell anemia. Ann Emerg Med. 1986; 15:787-91. [PubMed: 3729099]

17. Powers RD. Management protocol for sickle-cell disease patients with acute pain: impact on emergency department and narcotic use. Am J Emerg Med. 1986; 4:267-8. [PubMed: 3964371]

18. Benjamin, LJ.; Dampier, CD.; Jacox, A., et al. Quick reference guide for emergency department clinicians. American Pain Society; Glenview IL: 2001. Guideline for the Management of Acute Pain in Sickle-Cell Disease.

19. National Institutes of Health Heart, Lung, and Blood Diseases. [Accessed Jan 21, 2012] The management of sickle cell disease. Available at: http://www.nhlbi.nih.gov/health/prof/blood/ sickle/sc_mngt.pdf

20. Sickle Cell Society. Standards for the clinical care of adults with sickle cell disease in the United Kingdom. Sickle Cell Society; London, UK: 2008.

21. Platt OS, Brambilla DJ, Rosse WF, et al. Mortality in sickle cell disease. Life expectancy and risk factors for early death. N Engl J Med. 1994; 330:1639-44. [PubMed: 7993409]

22. Arendts G, Fry M. Factors associated with delay to opiate analgesia in emergency departments. J Pain. 2006; 7:682-6. [PubMed: 16942954]

23. Hick JL, Nelson SC, Hick K, Nwaneri MO. Emergency management of sickle cell disease complications: review and practice guidelines. Minn Med. 2006; 89(2):42-4. 47. [PubMed: 16700347]

24. American Pain Society. Principles of analgesic use in the treatment of acute pain and cancer pain. American Pain Society; Glenview IL: 2008. 


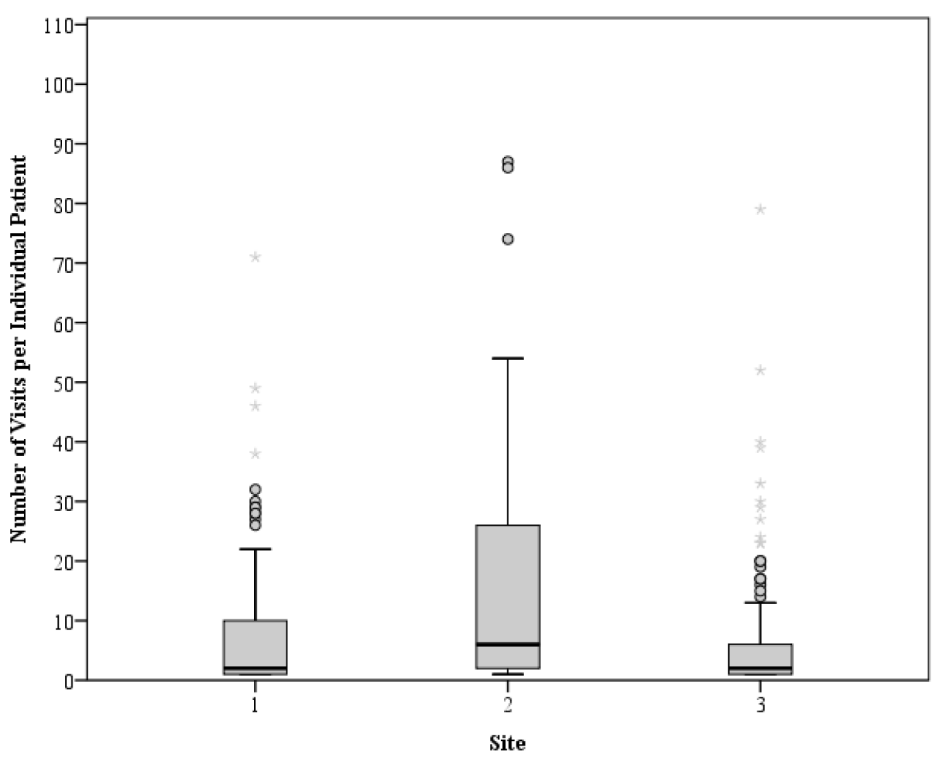

Figure1. Number of Visits per Individual Patient by Study Site

The boxplots represent the variability in the number of visits per individual patient at each site. The solid line represents the median, and the upper and lower ends of the "box" represent the $25^{\text {th }}$ and $75^{\text {th }}$ percentiles. The outer bars represent the $10^{\text {th }}$ and $90^{\text {th }}$ percentiles. 

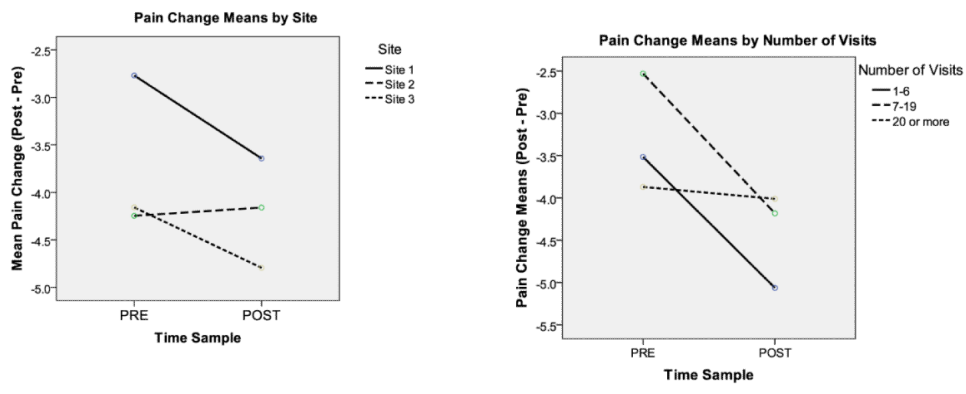

Figure 2. A \& B: Differences in Arrival to Discharge Pain Scores: Pre- and Post-Implementation of Analgesic Protocols

Patients at Site 1 and patients with fewer than 20 visits over the study period had the greatest changes from arrival to discharge pain scores between the pre- and post-implementation study periods. 


\section{Table 1}

Patient sample characteristics by site

\begin{tabular}{|lccc|}
\hline Characteristic & Site 1 & Site 2 & Site 3 \\
\hline Number of individual patients & 99 & 31 & 212 \\
Age, mean $( \pm$ SD) & $35( \pm 11)$ & $31( \pm 10)$ & $31( \pm 10)$ \\
Female, n $(\%)$ & $50(51)$ & $18(58)$ & $129(61)$ \\
Black or African American, n (\%) & $97(99)$ & $31(100)$ & $206(98.6)$ \\
\hline
\end{tabular}


Table 2

Univariate Differences in Time (Minutes) to Administration of Initial Analgesic: Pre- and PostImplementation of Analgesic Protocols

\begin{tabular}{|c|c|c|c|c|c|}
\hline Variable & $\begin{array}{c}\text { N: (Pre- } \\
\text { Implementa } \\
\text { tion Period) }\end{array}$ & $\begin{array}{l}\text { Pre Median } \\
\quad(\text { IQR) }\end{array}$ & $\begin{array}{c}\text { N: (Post- } \\
\text { Impleme } \\
\text { ntation } \\
\text { Period) }\end{array}$ & $\begin{array}{l}\text { Post Median } \\
\text { (IQR) }\end{array}$ & $\begin{array}{c}\text { Median } \\
\text { Difference } \\
(95 \% \text { CI })\end{array}$ \\
\hline \multicolumn{6}{|c|}{ Site Differences } \\
\hline Overall & 726 & 76 (49 to 139$)$ & 2147 & 92 (56 to 159$)$ & $10(6$ to 15$)$ \\
\hline Site 1 & 296 & 75 (48 to 138$)$ & 613 & $86(55$ to 128$)$ & $5(-3$ to 12$)$ \\
\hline Site 2 & 233 & 62 (44 to 88$)$ & 571 & 67 (45 to 101$)$ & $6(1$ to 12$)$ \\
\hline Site 3 & 197 & 143 (68 to 254$)$ & 963 & 127 (73 to 244$)$ & $-5(-20$ to 10$)$ \\
\hline \multicolumn{6}{|c|}{$\begin{array}{l}\text { ED Visits/Patient } \\
\text { Groups }\end{array}$} \\
\hline Overall & 726 & 76 (49 to 139$)$ & 2147 & 92 (56 to 159$)$ & $10(6$ to 15$)$ \\
\hline $1-6$ & 114 & $102(55$ to 201$)$ & 396 & 112 (64 to 202 ) & 5 (-11 to 22$)$ \\
\hline $7-19$ & 156 & $92(51$ to 166$)$ & 448 & 106 (68 to 192 ) & $17(4$ to 30$)$ \\
\hline $20+$ & 456 & $69(48$ to 121$)$ & 1303 & $84(53$ to 133$)$ & $9(4$ to 15$)$ \\
\hline
\end{tabular}

Among all sites, there was a slight increase in the time to administration of the initial analgesic from the pre- to post-implementation period. This was significant across patients regardless of the number of visits $(\mathrm{p}<0.001$, independent-samples median test, Hodges-Lehman statistic). 
Table 3

Summary of Inferential Tests for factorial ANOVA models predicting Time to Analgesic

\begin{tabular}{|lcc|}
\hline & Model 1 & Model 2 \\
\hline Source & $\mathrm{F}(\mathrm{p})$ & $\mathrm{F}(\mathrm{p})$ \\
Study Period & $0.14(0.702)$ & $0.07(0.788)$ \\
Site & $184.92(<0.001)$ & $229.68(<0.001)$ \\
Site $\times$ Study Period & $0.64(0.525)$ & \\
Visit & & $0.24(0.788)$ \\
Visit $\times$ Study Period & & $0.64(0.528)$ \\
\hline
\end{tabular}

Differences in the increase in time to administration of the initial analgesic from pre- to post-implementation period were due to increases in times at Site 2. 
Table 4

Univariate Differences in Arrival to Discharge Pain Scores: Pre- and Post-Implementation of Analgesic Protocols

\begin{tabular}{|c|c|c|c|c|c|}
\hline Variable & $\begin{array}{l}\text { N Pre- } \\
\text { protocol } \\
\text { period }\end{array}$ & $\begin{array}{l}\text { Pre Median } \\
\text { (IQR) }\end{array}$ & $\begin{array}{l}\text { N post- } \\
\text { protocol } \\
\text { period }\end{array}$ & $\begin{array}{l}\text { Post Median } \\
\text { (IQR) }\end{array}$ & $\begin{array}{l}\text { Median } \\
\text { difference } \\
(95 \% \mathrm{CI})\end{array}$ \\
\hline \multicolumn{6}{|c|}{ Site Differences } \\
\hline Overall & 295 & $-4(-5$ to -2$)$ & 458 & $-4(-6$ to -2$)$ & $-1(-1$ to 0$)$ \\
\hline Site 1 & 126 & $-2(-4$ to -1$)$ & 126 & $-4(-5$ to -2$)$ & $-1(-2$ to 0$)$ \\
\hline Site 2 & 150 & $-4(-6$ to -3$)$ & 269 & $-4(-5$ to -3$)$ & $0(0$ to 1$)$ \\
\hline Site 3 & 19 & $-4(-6$ to -3$)$ & 63 & $-5(-7$ to -3$)$ & $-1(-2$ to 1$)$ \\
\hline \multicolumn{6}{|c|}{ ED Visits/Patient Groups } \\
\hline Overall & 295 & $-4(-5$ to -2$)$ & 458 & $-4(-6$ to -2$)$ & $-1(-1$ to 0$)$ \\
\hline $1-6$ & 31 & $-3(-5$ to -1$)$ & 32 & $-5(-8$ to -2$)$ & $-1.5(-3$ to 0$)$ \\
\hline $7-19$ & 49 & $-2(-4$ to -1$)$ & 55 & $-4(-6$ to -2$)$ & $-2(-3$ to -1$)$ \\
\hline $20+$ & 215 & $-4(-6$ to -2$)$ & 371 & $-4(-5$ to -2$)$ & $0(-1$ to 0$)$ \\
\hline
\end{tabular}

There was a significant decrease in the pain score from arrival within 45 minutes to discharge patients between the pre- and post-implementation study periods, $(\mathrm{p}=0.021$, independent-samples median test, Hodges-Lehman statistic). 
Table 5

Summary of inferential tests for factorial ANOVA models predicting change in pain

\begin{tabular}{|lcc|}
\hline & Model 3 & Model 4 \\
\hline Source & $\mathrm{F}(\mathrm{p})$ & $\mathrm{F}(\mathrm{p})$ \\
Study Period & $3.51(0.062)$ & $16.34(<0.001)$ \\
Site & $14.39(<0.001)$ & \\
Site $\times$ Study Period & $3.012(0.0498)$ & \\
Visit & & $3.29(0.038)$ \\
Visit $\times$ Study Period & & $5.54(0.004)$ \\
\hline
\end{tabular}

Patients from Site 1, and patients with fewer visits experienced the largest decrease in pain scores from the pre- to the post-implementation period; the largest effect was seen in patients treated at Site 1. 
Table 6

Differences in Analgesic Agent Use and Route of Administration, Pre- and Post-Implementation of Analgesic Protocols, and Between Study Sites

\begin{tabular}{|c|c|c|c|}
\hline Variable & Pre $(\%)$ & Post (\%) & $P$ value \\
\hline \multicolumn{4}{|c|}{ Hydromorphone } \\
\hline Overall & 46.3 & 58.0 & $<0.001$ \\
\hline Site 1 & 71.9 & 63.0 & 0.008 \\
\hline Site 2 & 20.0 & 42.2 & $<0.001$ \\
\hline Site 3 & 38.8 & 64.1 & $<0.001$ \\
\hline \multicolumn{4}{|c|}{ Hydromorphone and NSAIDS } \\
\hline Overall & 1.1 & 4.3 & $<0.001$ \\
\hline Site 1 & 1.0 & 11.3 & $<0.001$ \\
\hline Site 2 & 1.3 & 0.2 & NS \\
\hline Site 3 & 1.0 & 2.1 & NS \\
\hline \multicolumn{4}{|c|}{ Morphine Sulfate } \\
\hline Overall & 41.7 & 29.3 & $<0.001$ \\
\hline Site 1 & 24.1 & 20.4 & NS \\
\hline Site 2 & 53.5 & 53.8 & NS \\
\hline Site 3 & 54.6 & 20.5 & $<0.001$ \\
\hline \multicolumn{4}{|l|}{ Oral } \\
\hline Overall & 2.5 & 4.3 & 0.028 \\
\hline Site 1 & 2.7 & 3.1 & 0.735 \\
\hline Site 2 & 0.4 & 0.4 & NS \\
\hline Site 3 & 4.6 & 7.4 & NS \\
\hline \multicolumn{4}{|c|}{ Intravenous } \\
\hline Overall & 65.6 & 60.8 & 0.021 \\
\hline Site 1 & 58.9 & 54.0 & NS \\
\hline Site 2 & 98.3 & 90.2 & $<0.001$ \\
\hline Site 3 & 37.1 & 47.4 & 0.007 \\
\hline \multicolumn{4}{|c|}{ Intramuscular } \\
\hline Overall & 28.9 & 29.4 & 0.786 \\
\hline Site 1 & 32.0 & 29.7 & NS \\
\hline Site 2 & 0.9 & 8.1 & $<0.001$ \\
\hline Site 3 & 57.4 & 41.9 & $<0.001$ \\
\hline \multicolumn{4}{|c|}{ Subcutaneous } \\
\hline Overall & 2.8 & 5.1 & 0.008 \\
\hline Site 1 & 6.4 & 13.2 & 0.002 \\
\hline Site 2 & 0.4 & 1.4 & NS \\
\hline Site 3 & 0 & 2.2 & 0.036 \\
\hline
\end{tabular}

NSAIDS = non-steroidal anti-inflammatory drugs; NS = not significant

There was an overall decrease in the use of morphine sulfate and increase in the use of hydromorphone. Sites 2 and 3 had the largest increase in the use of hydromorphone. 